\title{
Generating Shear Flows without Moving Parts by Thermo-Osmosis in Heterogeneous Nanochannels
}

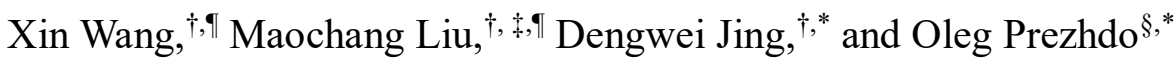

${ }^{\dagger}$ State Key Laboratory of Multiphase Flow in Power Engineering, Xi' an Jiaotong University Xi'an 710049, China

¿Suzhou Academy of Xi'an Jiaotong University, Suzhou, Jiangsu 215123, China

${ }^{\S}$ Department of Chemistry, University of Southern California, Los Angeles, CA 90089, USA

"These authors contributed equally to this work.

*To whom correspondence should be addressed. E-mail: dwjing@mail.xjtu.edu.cn, prezhdo@usc.edu. 


\section{Required time to reach steady flow state}

The total time we use for each simulation is $1,300,000$ time steps including initial 300,000 time steps for the system to reach steady state and subsequent $1,000,000$ time steps to collect related statistical data. Each time step is $0.005 \tau$. Using the typical value $\tau \sim 2 \mathrm{ps}$, just 3,000ps (3ns) is used in order to generate steady flows, which is quite short but enough due to the very small scale for nanofluidics as we will discuss below.

Theoretically, the needed time $\tau_{s}$ to get steady flows can be estimated by following expression $^{1}$

$$
\tau_{s} \sim \frac{\rho L^{2}}{\eta}
$$

where $\rho$ and $\eta$ are density and viscosity of fluid, respectively and $L$ can be chosen as the length of nanochannel in the direction parallel to the flow direction. Using $\rho \sim 0.8 / \sigma^{3}, \eta \sim 1.87 \varepsilon \tau / \sigma^{3}$ and $L \sim 27.2 \sigma$, the estimated $\tau_{s}$ is $\sim 320 \tau \sim 640 \mathrm{ps} \sim 64,000$ time steps. Accordingly, the time we use is sufficiently long for the system to reach steady flows theoretically.

Furthermore, to verify the theoretical estimation, the locations of the pistons have been recorded during the whole simulation instead of the temperature profiles in our work. Because there would be a steady net fluid transport between the two fluid reservoirs when the flows are steady, for which only the steady temperature gradients could account. It thus becomes simple to judge whether the flows are steady by monitoring the locations evolution of the pistons over simulation time.

The obtained results for fixed $\varepsilon_{\text {bottom-fluid }}=0.5 \varepsilon$ and varied $\varepsilon_{\text {bottom-fluid }}$ are shown in Figure S1. As we illustrate in the manuscript, the pistons are fixed in the initial 200,000 time steps $(\sim 1,000 \tau \sim 2,000 \mathrm{ps})$ for the system relaxation under stable thermal state. Then pistons are allowed to move freely in $\mathrm{x}$-direction for another 100,000 time steps $(\sim 500 \tau \sim 1,000 \mathrm{ps})$ and external forces are applied on them to keep the pressure on the two fluid reservoirs at desired value and to guarantee no pressure difference between the two fluid reservoirs. Thus, the fluid flow would only be resulted from the thermo-osmosis. During this period, the locations of piston are fluctuated sharply and then get stable quite fast. After the initial $1,500 \tau(\sim 3,000 \mathrm{ps})$, labelled as vertical black dash line, the locations of right and left pistons over time are nearly linear. Here, affected by the thermal noise, the curves show slight fluctuations. It indicates that 
the flows are stable and the system has reached stable state. Accordingly, available statistical data can be collected.

\section{Derjaguin expression for thermo-osmosis}

Derjaguin has related the flow direction of thermo-osmosis with the effective excess surface enthalpy based on the irreversible thermodynamics ${ }^{2}$. The derivation is shown below.

As a start, we consider a nanochannel connected by two fluid reservoirs filling with a singlecomponent fluid, as shown in Figure 2. To include a stable pressure gradient $\nabla P$ and a stable temperature gradient $\nabla T$ across the nanochannel imposed by the two fluid reservoirs, the entropy production rate $\psi$ is

$$
\psi=Q \nabla P+W \frac{\nabla T}{T}
$$

where $Q$ is the fluid volume flux and $W$ is the heat transfer flux. The transport of this fluid system can be characterized by the response matrix

$$
\left(\begin{array}{c}
Q \\
W
\end{array}\right)=\left(\begin{array}{ll}
\beta_{11} & \beta_{12} \\
\beta_{21} & \beta_{22}
\end{array}\right)\left(\begin{array}{c}
-\nabla P \\
-\frac{\nabla T}{T}
\end{array}\right)
$$

where $\beta_{i j}$ are phenomenological coefficients. The coefficients $\beta_{11}$ describes the isothermal mass transport driven by a pressure gradient and $\beta_{22}$ describes the isobaric heat transport by a temperature gradient. What we concern are the coefficient $\beta_{12}$, also called as the thermoosmosis coefficient, which describes the isobaric mass transport driven by a temperature gradient, as well as the coefficient $\beta_{21}$, which describes the isothermal heat transport by a pressure gradient. According to Onsager reciprocal relations, $\beta_{12}=\beta_{21}$.

To deduce $\beta_{21}$, only a pressure gradient $\nabla P$ instead of a temperature gradient $\nabla T$ is considered in the system.

$$
W=-\beta_{21} \nabla P
$$

The heat flux across the nanochannel, such as section $\mathrm{AA}^{\prime}$, is

$$
W_{1}=H_{B} Q+\int_{-h}^{h} \Delta H(y) v(y) d y
$$

The heat flux across the fluid reservoir, such as section BB', is

$$
W_{2}=H_{B} Q
$$


The excess heat flux driven by a pressure gradient in the nanochannel is

$$
W=W_{1}-W_{2}=-\beta_{21} \nabla P=\int_{-h}^{h} \Delta H(y) v(y) d y
$$

For the case where the nanochannel height is much larger than the thickness interfacial layer $\mathrm{h}_{\mathrm{s}}$, the velocity profiles within the bottom and upper interfacial layers can be assumed linear with the non-slip boundary conditions

$$
\mathrm{v}(\mathrm{y})=\frac{-2 \mathrm{~h}(\mathrm{y} \pm \mathrm{h}) \nabla \mathrm{P}}{\eta}
$$

Considering only the bottom half of the nanochannel, eq 7 can be written as

$$
\beta_{12}=\beta_{21}=\frac{1}{\eta} \int_{0}^{h_{s}} \Delta H(y) y d y
$$

We introduce the effective excess enthalpy $\overline{\Delta H}$, which is assumed to be distributed uniformly within the interfacial layer, so

$$
\beta_{12}=\frac{h_{s}^{2} \overline{\Delta H}}{2 \eta}
$$

Accordingly, the sigh of coefficients $\beta_{12}$, which dictates the direction of thermo-osmosis flows, are associated with the effective enthalpy $\overline{\Delta \mathrm{H}}$.

\section{Effect of nanochannel height on shear rates}

The height of the nanochannel is expected to affect the shear flows. Theoretically, the velocities near the surfaces would be determined by thermo-osmosis. For fixed temperature gradients and wetting surfaces, the velocities near the upper and bottom walls would be decided. Therefore, the shear rates are expected to decrease linearly with the increased heights of nanochannel. However, we find the simulation results are slightly different from the theoretical prediction. As shown in Figure S3, the thermo-osmosis velocities near the bottom surfaces are not fixed and show a decline trend with the decreased heights. We suggest this decline is caused by the viscous drag imposed by velocities near the upper surfaces. With the reduced nanochannel height, the velocities near the upper surfaces would have a greater effect on the velocities near the bottom surfaces. Overall, with the increased nanochannel height the shear 
rates decrease.

It should be noted that the lower limit of the height is chosen according to the fluid enthalpy distributions. As shown in Figure 3(b) in the manuscript, the thickness of interfacial layer, where the enthalpy is different from the bulk value, is around $6 \sigma$. We should avoid the overlap of the upper and bottom interfacial layers. Since this may render the results complex and hard to explain, which has been reported by $\mathrm{Fu}$ et $\mathrm{al}^{3}$. Accordingly, the height of the nanochannel should be larger than $12 \sigma$ to avoid the overlap of interfacial layers.

\section{Estimation of Reynolds number}

Using the values of bulk fluid $\rho \sim 0.8 / \sigma^{3}$ and $\eta \sim 1.87 \varepsilon \tau / \sigma^{3}$, the height of the nanochannel $h \sim 20.41 \sigma$, and the maximum velocity in our work $v \sim 0.02 \sigma / \tau$, we can obtain the maximum Reynolds number $R e=\rho v h / \eta \sim 0.17$, which is quite small. The flow is in the form of laminar flow. It indicates that Reynolds number can be neglected in nanofluidics despite its significance at macroscale. Accordingly, there is no need to analyze the results based on Reynolds number. 


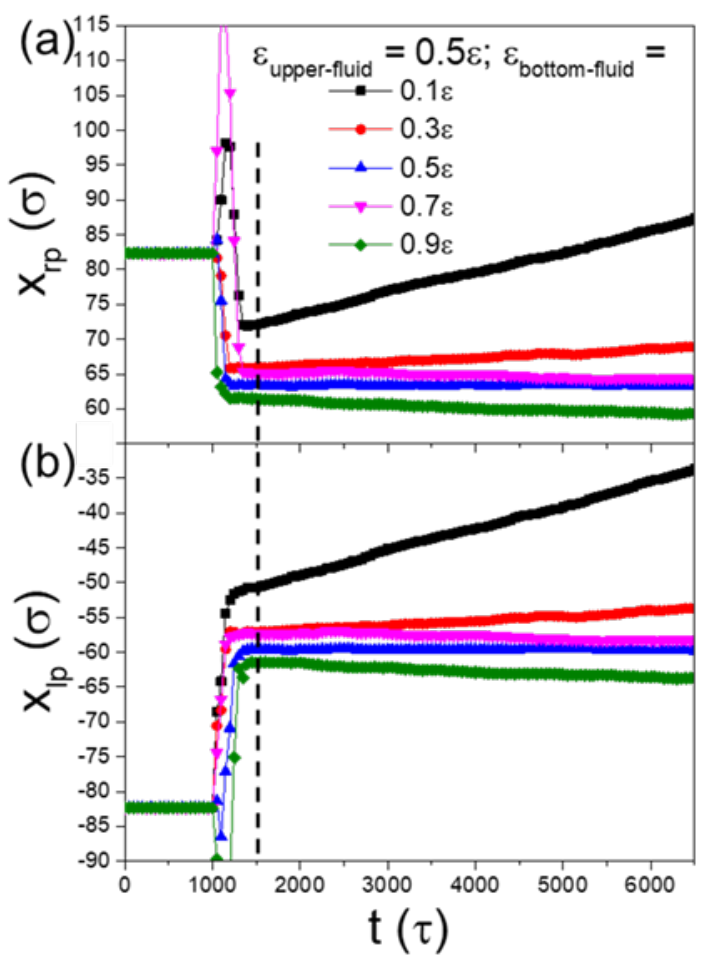

Figure S1. The locations of (a) right piston $x_{r p}$ and (b) left piston $x_{l p}$ in x-direction as a function of time for fixed $\varepsilon_{\text {upper-fluid }}=0.5 \varepsilon$ and varied $\varepsilon_{\text {bottom-fluid. }}$ 


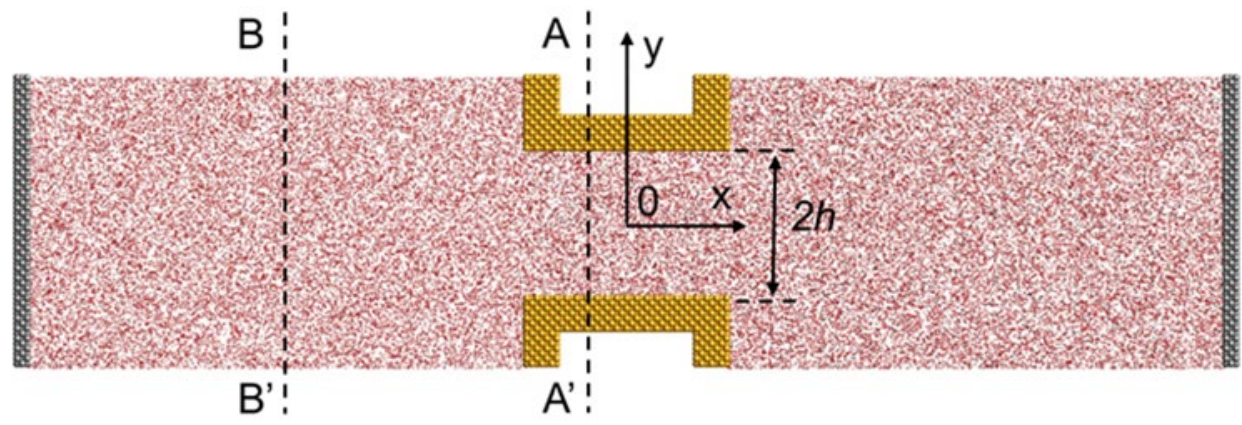

Figure S2. Schematic for the derivation of thermo-osmosis coefficients. 

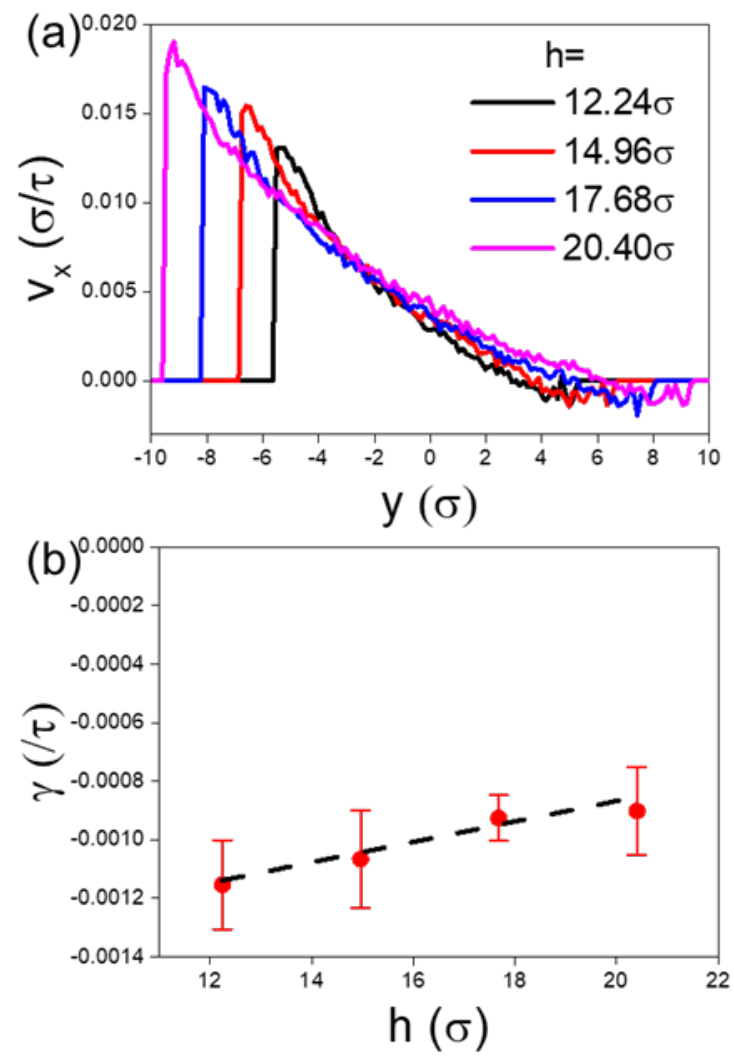

Figure S3. (a) Velocity profiles in the flow direction $v_{x}$ inside the nanochannel and (b) shear rates $\gamma$ for $\varepsilon_{\text {upper- }}$ fluid $=0.9 \varepsilon$ and $\varepsilon_{\text {bottom-fluid }}=0.1 \varepsilon$ under varied heights of nanochannel under the temperature difference $\Delta \mathrm{T}=$ $0.2 \varepsilon / \mathrm{k}_{\mathrm{b}}$. 


\section{References}

1. Squires, T. M.; Quake, S. R. Microfluidics: Fluid Physics at the Nanoliter Scale. Rev. Mod. Phys. 2005, 77, 977.

2. Derjaguin, B. V.; Churaev, N. V.; Muller, V. M.; Kisin, V. Surface Forces; Springer, 1987.

3. Fu, L.; Merabia, S.; Joly, L. Understanding Fast and Robust Thermo-Osmotic Flows through Carbon Nanotube Membranes: Thermodynamics Meets Hydrodynamics. J. Phys. Chem. Lett. 2018, 9, 2086-2092. 